\title{
Convulsions in a 17-year-old boy after a head injury sustained while playing hockey
}

\author{
Michael J. Ellis MD, Richard Wennberg MD PhD
}

A 17-year-old boy was playing hockey at an outdoor rink when his skate caught a rut in the ice and he fell backward, striking the back of his head on the ice. He was not wearing a helmet. Eyewitnesses reported that the patient lost consciousness upon impact, with convulsions involving his arms and legs that lasted about 90 seconds. No evidence of lateralizing signs, tongue-biting or urinary incontinence was seen.

The patient was transported by ambulance to a local emergency department where he underwent computed tomography (CT) of the head that showed right parietal swelling of the scalp. There was no skull fracture or intracranial hemorrhage. Radiography of the cervical spine was completed and did not show any abnormalities. After overnight admission to hospital for observation, the patient was referred to a multidisciplinary pediatric concussion program for further evaluation and follow-up.

The patient was seen in consultation seven days later, at which time he complained of mild headache and trouble falling asleep. His medical history was unremarkable except for mild asthma. He had no personal or family history of seizures. A neurologic examination was normal. Concussive convulsions were diagnosed.

In keeping with the diagnosis, no antiepileptic medication was prescribed, and the patient was provided appropriate return-to-learn and return-to-play counselling. After successfully completing a graduated return-to-play protocol, the patient was safely returned to full-contact hockey without complication 21 days after his injury. The patient's driver's licence was not suspended, and he was advised to wear a helmet during future hockey activities.

\section{Discussion}

Sports-related concussion is a form of traumatic brain injury that is caused by the transmission of abnormal biomechanical forces to the brain resulting in temporary alterations in neurological functioning. ${ }^{1}$ Convulsions occurring at the time of sports-related concussion are uncommon and can present a substantial diagnostic and management challenge for physicians who are unfamiliar with the nature of these events. For these patients' cases, optimizing management requires physicians to distinguish between concussive convulsions and posttraumatic seizures or epilepsy - conditions that differ considerably in terms of pathophysiology, natural history and treatment recommendations.

\section{Concussive convulsions}

Concussive convulsions are characterized by myoclonic or tonic-clonic motor convulsions that occur within seconds of head injury. They are thought to occur in the absence of epileptic brain activity. Initially described in adults by Jennet $^{2}$ as "immediate epilepsy" — defined explicitly as the occurrence of a generalized convulsion "within moments of injury" - the phenomenon was found to have no association with the development of subsequent seizures. ${ }^{2}$

Much of what has since been learned about concussive convulsions is derived from observational studies involving elite adult Australian rules football and rugby league players, where the reported incidence of concussive convulsions is about 1 in every 70 cases of concussion. ${ }^{3}$ In a retrospective study, McCrory and colleagues ${ }^{4}$ detailed the cases of 22 adult football players with concussive convulsions. Within two seconds of head impact, the athletes typically displayed a loss

\section{KEY POINTS}

- Concussive convulsions are nonepileptic events that have a benign natural history.

- In the setting of a reliable clinical history, advanced neuroimaging and electroencephalgraphy are not required.

- Antiepileptic medication is not indicated, and physicians should focus their attention on management of the associated concussion and optimization of preventive education.
Competing interests: None declared.

This article has been peer reviewed.

The authors have obtained

Correspondence to: Michael Ellis, mellis3@panamclinic.com

CMAJ 2016. DOI:10.1503 /cmaj.150124 patient consent. 
of consciousness and brief tonic phase, followed by bilateral myoclonic or clonic jerking of the extremities lasting no longer than 150 seconds. Following the event, patients showed clinical symptoms consistent with sports-related concussion. All players underwent neuroimaging studies with normal results. Twenty-one players underwent electroencephalography (EEG), which was normal in all cases, with the exception of one player who showed transient left temporal slow wave activity in the period directly after injury and in whom EEG studies were normal in followup at one week and two years. All of the athletes returned to full sporting activities, and none experienced recurrent seizures. The authors postulated that concussive convulsions were not caused by structural brain injury and the pathophysiological mechanisms governing posttraumatic seizures, but were instead likely mediated by transient functional decerebration similar to the corticomedullary dissociation seen in convulsive syncope. ${ }^{3,4}$

Since these seminal studies, a subsequent prospective study involving Australian footballers found that more subtle motor manifestations, particularly tonic posturing without clonic movements, occurred in almost a quarter of sportsrelated concussions, likely representing a less extreme form of the concussive convulsion phenomenon with an equally benign outcome. ${ }^{5}$

Current practice recommendations suggest that neuroimaging and EEG studies have normal results in this patient population ${ }^{3}$ and should only be used to rule out structural brain injury if other clinical indications are present, such as focal neurologic deficit or altered level of consciousness. ${ }^{1}$ The prognosis of this condition is excellent, and antiepileptic medication is not required. Patients who have concussive convulsions can be safely returned to full sporting activities after documented recovery from the associated concussion.

\section{Posttraumatic seizures}

In contrast to concussive convulsions, posttraumatic seizures are characterized by acute physical manifestations of epileptic brain activity occurring in patients after a traumatic brain injury. Diagnostically, the term posttraumatic seizure is used to define a single epileptic event; patients who have recurrent, unprovoked seizures after a traumatic brain injury receive a diagnosis of posttraumatic epilepsy, a condition affecting $7 \%-57 \%$ of all patients with traumatic brain injury. ${ }^{6}$

Posttraumatic seizures are further classified based on the timing of the event, defined as early (less than one week after injury) and late (more than one week after injury). ${ }^{6}$ Any seizure within 24 hours of traumatic brain injury, with the sole exception of a convulsion occurring within seconds of the injury, should be considered an early posttraumatic seizure, and not a concussive convulsion.

The pathophysiology and clinical presentation of posttraumatic seizures are strongly related. Early posttraumatic seizures are thought to represent epileptic phenomena caused by direct injury to brain tissue. Late posttraumatic seizures and posttraumatic epilepsy are proposed to be mediated by a progressive cellular and molecular process of local "epileptogenesis." In contrast to concussive convulsions that present with stereotypic clinical manifestations, the signs of posttraumatic seizures are dependent on the location of brain injury and can manifest as focal seizures, generalized seizures or focal seizures with secondary generalization. The natural history of early posttraumatic seizures is generally more favourable than that of late posttraumatic seizures, but both carry a substantial risk of developing into posttraumatic epilepsy. ${ }^{7}$

Although evidence-based guidelines exist for the evaluation and management of a single unprovoked seizure in children and adolescents, ${ }^{8,9}$ similar guidelines specific to patients with a single seizure that occurred after a traumatic event are lacking. Evaluation of patients with a posttraumatic seizure includes consideration of neuroimaging and EEG to identify the location and electrical behaviour of the associated structural brain injury. Magnetic resonance imaging is the neuroimaging modality of choice for non-urgent evaluation of patients with suspected lesional epilepsy, ${ }^{8}$ offering superior contrast resolution and the ability to incorporate sequences, such as susceptibility-weighted imaging, with enhanced sensitivity to cerebral microhemorrhages.

Although case-control studies have shown that treatment of a first unprovoked seizure does not affect long-term rates of seizure remission in children and adults, ${ }^{10}$ management of care in pediatric patients with posttraumatic seizures should be individualized. Adolescents and young adults with structural brain lesions and concordant epileptiform abnormalities on EEG may be at an elevated risk of recurrent seizures and should be considered for treatment with antiepileptic medications and immediate suspension of driving privileges. ${ }^{9}$ For such patients, long-term follow-up and monitoring by an experienced neurologist is suggested.

\section{Conclusion}

The evaluation of patients presenting with convulsions after a head injury requires the physician to distinguish between concussive convulsions and posttraumatic seizures. A careful 
clinical history and familiarity with the available evidence can help guide care for these patients. In the setting of a clear history of concussive convulsions, neuroimaging and EEG studies are not required, and treatment with antiepileptic medication is not indicated. Physicians should advise patients of the benign natural history of these events and focus their attention on the management of the patient's associated concussion and optimization of preventive education.

\section{References}

1. McCrory P, Meeuwisse WH, Aubry M, et al. Consensus statement on concussion in sport: the 4th International Conference on Concussion in Sport held in Zurich, November 2012. Br J Sports Med 2013;47:250-8.

2. Jennet B. Epilepsy after non-missile head injuries. Chicago: Heinemann; 1975:67.

3. McCrory PR, Berkovic SF. Concussive convulsions. Incidence in sport and treatment recommendations. Sports Med 1998;25: 131-6.

4. McCrory PR, Bladin PF, Berkovic SF. Retrospective study of concussive convulsions in elite Australian rules and rugby league footballers: phenomenology, aetiology, and outcome. BMJ 1997;314:171-4

5. McCrory PR, Berkovic SF. Video analysis of acute motor and convulsive manifestations in sport-related concussion. Neurology 2000;54:1488-91.

6. Hunt RF, Boychuk JA, Smith BN. Neural circuit mechanisms of post-traumatic epilepsy. Front Cell Neurosci 2013;7:89.

7. Lamar CD, Hurley RA, Rowland JA, et al. Post-traumatic epilepsy: review of risks, pathophysiology, and potential biomarkers. J Neuropsychiatry Clin Neurosci 2014;26:iv-113.

8. Hirtz D, Ashwal S, Berg A, et al. Practice parameter: evaluating a first nonfebrile seizure in children: report of the quality standards subcommittee of the American Academy of Neurology, The Child Neurology Society, and The American Epilepsy Society. Neurology 2000;55:616-23.

9. Hirtz D, Berg A, Bettis D, et al. Practice parameter: treatment of the child with a first unprovoked seizure: Report of the Quality Standards Subcommittee of the American Academy of Neurology and the Practice Committee of the Child Neurology Society. Neurology 2003;60:166-75.

10. Beghi E. Management of a first seizure. General conclusions and recommendations. Epilepsia 2008;49(Suppl 1):58-61.

Affiliations: Department of Surgery and Pediatrics and Child Health (Ellis), Section of Neurosurgery, University of Manitoba, Pan Am Concussion Program, Winnipeg, Man.; Department of Neurology (Wennberg), University of Toronto, Canadian Sport Concussion Project, Toronto, Ont.

Contributors: Michael Ellis and Richard Wennberg contributed to the conception of the manuscript. Michael Ellis drafted the original manuscript. Richard Wennberg critically revised the manuscript. Both authors approved the final version of the manuscript to be published and agree to act as guarantors of the work.

The section Cases presents brief case reports that convey clear, practical lessons. Preference is given to common presentations of important rare conditions, and important unusual presentations of common problems. Articles start with a case presentation (500 words maximum), and a discussion of the underlying condition follows (1000 words maximum). Visual elements (e.g., tables of the differential diagnosis, clinical features or diagnostic approach) are encouraged. Consent from patients for publication of their story is a necessity. See information for authors at www.cmaj.ca.

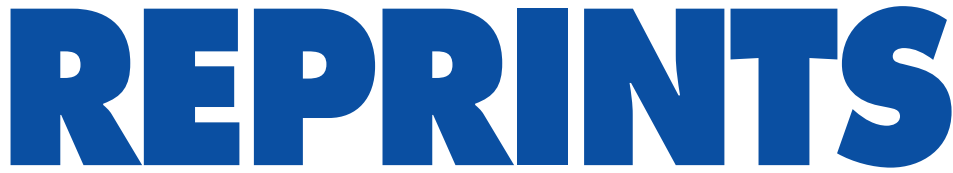

We have partnered with Sheridan Press!

To purchase commercial article reprints and e-prints or to request a quote, please contact

Matt Neiderer

Content Sales

Sheridan Content Services
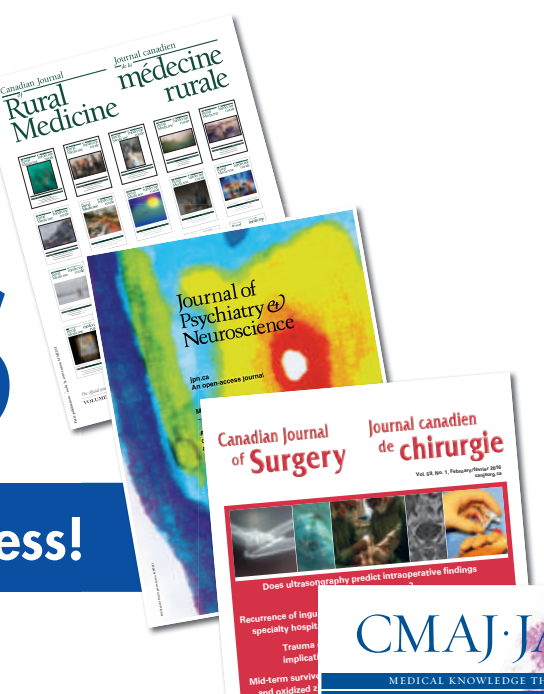

Recommendations on
screening for colorectal screening for colorectal
cancer in primary care cancer in primary cace
Who should be screened?
How oftern what screning
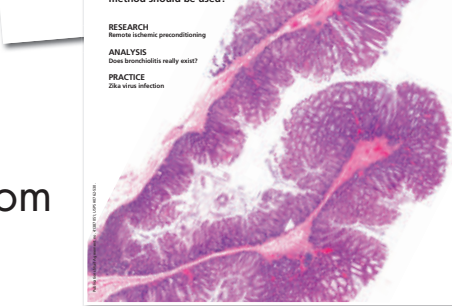